This item was submitted to Loughborough's Research Repository by the author.

Items in Figshare are protected by copyright, with all rights reserved, unless otherwise indicated.

\title{
Is the joint-angle specificity of isometric resistance training real? And if so, does it have a neural basis?
}

PLEASE CITE THE PUBLISHED VERSION

https://doi.org/10.1007/s00421-019-04229-z

PUBLISHER

Springer

VERSION

AM (Accepted Manuscript)

\section{PUBLISHER STATEMENT}

This is a post-peer-review, pre-copyedit version of an article published in European Journal of Applied Physiology. The final authenticated version is available online at: https://doi.org/10.1007/s00421-019-04229-z.

\section{LICENCE}

CC BY-NC-ND 4.0

\section{REPOSITORY RECORD}

Lanza, Marcel B., Tom Balshaw, and Jonathan Folland. 2019. "Is the Joint-angle Specificity of Isometric Resistance Training Real? and If So, Does It Have a Neural Basis?”. Loughborough University. https://hdl.handle.net/2134/12555365.v1. 


\section{TITLE PAGE}

\section{Title:}

Is the joint-angle specificity of isometric resistance training real? and if so does it have a neural basis?

\section{Authors:}

Lanza, M.B. ${ }^{1,2}$, Balshaw, T.G. ${ }^{1}$, Folland, J.P ${ }^{1}$.

\section{Affiliations:}

${ }^{1}$ School of Sport, Exercise, and Health Sciences, Loughborough University, Leicestershire, UK

${ }^{2}$ CAPES Foundation, Ministry of Education of Brazil, Brasilia - DF 70040-020, Brazil.

\section{Abbreviated title for running head:}

Joint-angle specificity after resistance training

\section{Corresponding author:}

Dr. Marcel Bahia Lanza

School of Sport, Exercise, and Health Sciences, Loughborough University, Leicestershire, UK, LE11 3 TU. Email: marcel.lanza@gmail.com.

Orcid: https://orcid.org/0000-0002-9898-3070 


\begin{abstract}
Purpose: There are suggestions that isometric resistance training (RT) produces highly angle specific changes in strength with the greatest changes at the training angle, but these effects remain controversial with limited rigorous evidence, and the possible underpinning physiological mechanism(s) remain opaque. This study investigated the extent of angle specific changes in strength and neuromuscular activation after RT in comparison to a control group. Methods: A RT group $(n=13)$ performed 14 isometric RT sessions at a kneejoint angle of $65^{\circ}\left(0^{\circ}\right.$ is anatomical position) over a 4-week period, whilst a control group $(\mathrm{CON}, \mathrm{n}=9)$ maintained their habitual activity. Pre- and post-test sessions involved voluntary and evoked isometric knee extension contractions at five knee-joint angles $\left[35^{\circ}, 50^{\circ}, 65^{\circ}, 80^{\circ}\right.$ and $\left.95^{\circ}\right]$ while electromyography was recorded. Results: RT group increased maximum voluntary torque (MVT) at the training angle $\left(65^{\circ} ;+12 \%\right)$ as well as $80^{\circ}(+7 \%), 50^{\circ}(+11 \%)$ and $35^{\circ}(+5 \%)$. Joint angle specificity was demonstrated within the RT group (MVT increased more at some angles vs. others), and also by more rigorous between group comparisons (i.e. larger improvements after RT vs. CON at some angles than others). For the RT group normalized EMG increased at 3 of the same joint angles as strength, but not for CON. Importantly, however, neither within- or between group analyses provided evidence of joint angle specific changes in activation. Conclusion: In conclusion this study provides robust evidence for joint angle specific strength gains after isometric RT, with weaker evidence that changes in neuromuscular activation may contribute to these adaptations.
\end{abstract}

\title{
Key words
}

Neuromuscular activation

Muscle contractile properties

Torque production

Angle Specificity 


\section{Abreviation}

$\mathrm{CON}$ - Control group

$\mathrm{CV}_{\mathrm{W}}$ - Within-participant coefficient of variation.

ECT - Explosive-contraction training

EMG - Electromyography.

EVC - Explosive voluntary contraction.

$\mathrm{M}_{\mathrm{MAX}}$ - supramaximal muscle compound action potential.

$\mathrm{M}_{\text {MAX }}$ P-P- $\mathrm{M}_{\text {MAX }}$ peak-to-peak amplitude.

MVC - Maximum voluntary contraction.

MVT - Maximum voluntary torque.

Octet PT - Octet peak torque.

Octet $\mathrm{T}_{50}$ - Octet torque measure at 50 milliseconds after torque onset.

QEMG $_{0-50}$ - Quadriceps femoris EMG epoch between 0 to 50 milliseconds after EMG onset.

QEMG $_{0-100}$ - Quadriceps femoris EMG epoch between 0 to 100 milliseconds after EMG onset.

QEMG $_{0-150}$ - Quadriceps femoris EMG epoch between 0 to 150 milliseconds after EMG onset.

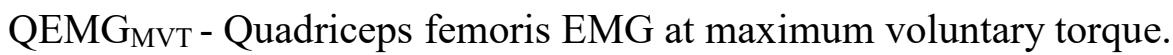

RF - Rectus femoris.

RT - Resistance Training

SCT - Sustained contraction training

$\mathrm{T}_{50}$ - Explosive torque at 50 milliseconds after torque onset.

$\mathrm{T}_{100}$ - Explosive torque at 100 milliseconds after torque onset.

$\mathrm{T}_{150}$ - Explosive torque at 150 milliseconds after torque onset.

Twitch PT - Twitch peak torque.

Twitch $\mathrm{T}_{50}$ - Twitch torque measure at 50 milliseconds after torque onset.

VL - Vastus lateralis.

VM - Vastus medialis. 


\section{Introduction}

Regular resistance training (RT) is widely recommended to improve muscle function (Folland and Williams 2007) and maintain musculoskeletal health (Morganti et al. 1995). Isometric RT and in particular sustained contraction training at high-force ( $\mathrm{SCT}, \geq 2 \mathrm{~s}$ duration; $>70 \%$ maximum force) and at one specific joint angle may result in highly angle specific changes in strength with the greatest changes at the training angle (Thepaut-Mathieu et al. 1988; Weir et al. 1995; Noorkoiv et al. 2014; Lum and Barbosa 2019). However evidence for these effects remains controversial with previous studies using the knee joint reporting a spectrum of responses from increases in strength only at the training angle [i.e. suggestive of angle specific changes; (Gardner 1963)] through to increases at the training angle and some but not all other angles [suggesting limited angle specificity; (Weir et al. 1995; Noorkoiv et al. 2014; Alegre et al. 2014)] and increases at all measured angles [no angle specificity (Kubo et al. 2006)]. Importantly though, rigorous demonstration of angle specificity effects requires not just changes at some angles and not others, but quantitative differences in joint angle responses (e.g. greater changes at one angle compared to another), although few studies have made these careful comparisons. In addition, use of a control group, which appears necessary to isolate the influence of a RT intervention from other potentially confounding factors [e.g. learning to perform the test (Kinser and Robins 2013)] or measurement issues such as imperfect calibration or imprecise replication of the joint positions) has been included in just two studies, but without rigorous between group statistical comparisons (Gardner 1963; Weir et al. 1995). Therefore robust statistical evidence for isometric RT producing changes in strength that are specific to the training angle (e.g. training angle vs. other angles) and the training intervention (e.g. RT vs. control group), and thus ultimately a group $\mathrm{x}$ angle interaction effect, are lacking. Hence, use of a control group and appropriate statistical procedures are necessary in order to fully assess whether joint angle specificity and the adaptations induced by isometric RT are genuine systematic effects.

The adaptations to short-term RT are typically ascribed primarily to neural adaptations, including increased neuromuscular activation of the agonist muscle (Gabriel et al. 2006; Folland and Williams 2007), and this has been proposed to be the mechanistic explanation for angle specific adaptations (Thepaut-Mathieu 
et al. 1988; Kitai and Sale 1989; Kubo et al. 2006; Noorkoiv et al. 2014). Similar to the disparate findings for joint angle specific torque changes, after RT neuromuscular activation has been assessed with surface electromyography (sEMG) and reported to: increase at a wide range of angles [up to $60^{\circ}$ from the training angle (Kubo et al. 2006)]; increase for some but not all angles proximal to the training angle [i.e. within $20^{\circ}$ of the training angle (Noorkoiv et al. 2014)]; and to remain unchanged at the training angle (Gardner 1963; Weir et al. 1995; Ullrich et al. 2009). Therefore, whether increases in agonist neuromuscular activation underpin joint angle specific strength increases after short-term SCT remains unclear. These contrasting findings may be due to a lack of contemporary sEMG methods such as joint angle specific M-wave normalization (Lanza et al. 2017), duplicate sensors on each superficial constituent muscle (Balshaw et al. 2017), and duplicate measurement sessions at each timepoint (i.e. pre and post training) to enhance measurement reliability.

The ability to produce torque as quickly as possible from a low/resting level, known as explosive torque, is important for explosive athletic performance (Paasuke et al. 2001; Tillin et al. 2013a) but also appears to be critical to recovering from a loss of balance and thus fall prevention (Izquierdo et al. 1999; Pijnappels et al. 2008; Behan et al. 2018). Previous research has shown marked training/contraction specific adaptations, such that explosive-contraction training (ECT), but not SCT, is effective at increasing early phase explosive torque $[\leq 100 \mathrm{~ms}$ after contraction onset (Tillin and Folland 2014; Balshaw et al. 2016)]. However, it is unknown if the addition of explosive-contractions to SCT may facilitate a broader range of adaptations with increases in both maximal and explosive strength.

Therefore, the primary aim of this study was to examine the extent of angle specific changes in strength after RT (i.e. greater increases at the training angle and in comparison, to a control group), and whether changes in neuromuscular activation might explain any angle specific strength changes. We hypothesized that the training intervention would increase strength at the training angle $\left(65^{\circ}\right)$ by more than the outermost angles $\left(35^{\circ}\right.$ and $\left.95^{\circ}\right)$ and in comparison to a control group (i.e. group $\mathrm{x}$ angle interaction), and that this would be explained by increases in neuromuscular activation. The secondary aim was to investigate if the addition of ECT to SCT facilitated improvements in early phase explosive strength, as well as the expected increases in maximum strength. 


\section{METHODS}

\section{Participants}

Twenty-two recreationally active males with no previous major lower-body injuries or systematic lower body RT for at least 12 months were recruited and randomly assigned to either a RT ( $n=13$; age, $22 \pm 3$ years; height, $1.78 \pm 0.07 \mathrm{~m}$; body mass, $73 \pm 7 \mathrm{~kg}$ ) or control group (CON; $n=9$; age, $23 \pm 3$ years; height, $1.79 \pm 0.08 \mathrm{~m}$; body mass, $75 \pm 8 \mathrm{~kg}$ ) and completed this study. This sample size was justified by a priori power analysis based on pilot work with a target effect size of 0.8 , alpha of 0.05 and power of $0.8(n=16)$. The power analysis calculations were performed by using the G*Power software (version 3.1.4; Heinrich Heine University, Düsseldorf, Germany). Ethical approval was granted by the Loughborough University Human Participants Sub-Committee and participants provided written informed consent prior to their participation according to the principles of The Declaration of Helsinki.

\section{Overview}

All training and testing of the knee extensors (unilateral contractions of the dominant leg) were performed at a consistent time of the day with participants seated and strapped to an adjustable custom-made isometric testing chair (Maffiuletti et al. 2016) at a constant hip-joint angle of $70^{\circ}\left(0^{\circ}\right.$ is the anatomical position). Participants first visited the laboratory for a familiarization session involving extensive practice of the voluntary contractions at the full range of angles, and experience with evoked twitch contractions. After familiarization, two duplicate laboratory measurement sessions were conducted both pre and post the 4-week intervention period (i.e. 4 measurement sessions in total). Pre-measurement sessions occurred 3-7 days apart prior to the first training session and post measurement sessions were performed 3 days after the last training session and then 2-3 days later. Each measurement session involved isometric knee extension contractions [maximum voluntary contractions (MVC), explosive voluntary contractions (EVC)], and evoked twitch contractions at five different knee-joint angles $\left[35^{\circ}, 50^{\circ}, 65^{\circ}, 80^{\circ}\right.$ and $95^{\circ}$ (where $0^{\circ}$ is anatomical position)]. 
Joint angles were tested in an opposite and counterbalanced order (Fig. 1) during both pre and both post measurement sessions. Torque and superficial quadriceps femoris EMG [vastus lateralis (VL), vastus medialis $(\mathrm{VM})$ and rectus femoris $(\mathrm{RF})]$, were recorded during maximum and explosive contractions, as well as evoked twitch contractions. Two octet contractions [8 pulses at $300 \mathrm{~Hz}$; known to drive the muscle at its maximum ability to produce explosive torque for $\sim 50 \mathrm{~ms}$ (de Ruiter et al. 2004)] were also evoked at each angle during one pre and one post measurement session. The RT group completed 14 training sessions in 4 weeks (x3/week for 2 weeks and then $\mathrm{x} 4 /$ week for 2 weeks) at a $65^{\circ}$ knee-joint angle, and each session involved a combination of ECT and SCT. The RT and CON groups were instructed to continue with their habitual physical activity and lifestyle, and CON only attended the pre- and post-measurement sessions.

\section{Training}

A brief warm-up of sub maximum contractions $[50 \%(\times 3), 75 \%(\times 2)$, and $90 \%(1 \times)$ counting a total duration of $\sim 3$ min including $30 \mathrm{~s}$ rest between each contraction] was performed before each RT session. Within each individual training session participants completed ECT (three sets of ten repetitions with $10 \mathrm{~s}$ between contractions; $\sim 1$ min per set) followed by SCT (three sets of six repetitions with a $30 \mathrm{~s}$ between contraction; $\sim 3$ min per set) of their dominant leg, with 2-min rest between each set and approximately 25 min per session. For the ECT repetitions participants were instructed to perform each contraction "as fast and hard as possible" up to $\geq 80 \%$ of maximum voluntary torque (MVT) for $\sim 1 \mathrm{~s}$, and then relax for $5 \mathrm{~s}$ between repetitions. A computer monitor was used to display peak rate of torque development (10-ms time epoch) to provide biofeedback of explosive performance. The torque-time curve was also shown: with a horizontal cursor at $80 \%$ MVT to ensure sufficiently forceful contractions; on a sensitive scale highlighting baseline torque to observe and correct any pre- tension or countermovement. For SCT each contraction was an MVC and participants were instructed to "push as hard and fast as possible" for $3 \mathrm{~s}$ with $20 \mathrm{~s}$ rest between contractions. During the MVCs a computer monitor was used to display a target torque trace $4 \mathrm{~s}$ before every contraction and participants were instructed to exceed the horizontal target line (i.e. the maximum torque 
achieved until that session) and verbal encouragement was provided. During each training session, the highest torque value achieved during an MVC at any point during the intervention up until that point (i.e. measurement or training session), was used to prescribe both ECT and SCT repetitions.

\section{Torque, surface EMG and video recording}

Participants were seated on an adjustable custom-made isometric knee extension dynamometer, with straps across the chest and waist to reduce extraneous body movement. This dynamometer is highly rigid with no padding and tight inextensible straps, and thus affords minimal movement between rest and MVC. The dynamometer configuration was established for each participant during familiarisation, and the actual knee joint angles at each position were assessed during this session with sagittal plane video images of the leg recorded during MVCs using a video camera placed lateral to the participant (Panasonic HC-V110, Secaucus, New Jersey, US). Knee-joint angle was determined as the angle between visible markers placed on the greater trochanter, lateral knee-joint space and lateral malleolus by digitising the video images using freely available public domain analysis software (Kinovea 0.8.15).

Force was measured with a calibrated S-beam strain-gauge (linear range 0-1500 N, Force Logic, Swallowfield, UK) which was attached perpendicular and posterior to the tibia with a reinforced inextensible webbing strap ( $35 \mathrm{~mm}$ width) fastened $\sim 3 \mathrm{~cm}$ superior to the lateral malleolus. Force was sampled and recorded at 2,000 $\mathrm{Hz}$ using an analogue-to-digital converter (A/D Micro 1401, CED, Cambridge, UK) and a computer utilising Spike 2 software (CED, Cambridge, UK). A $50 \mathrm{~Hz}$ notch filter with an infinite impulse response digital filter (q-factor of 10) was used to remove mains frequency noise and the force signal was also low-pass filtered at $500 \mathrm{~Hz}$ with a fourth-order zero-lag Butterworth digital filter. Torque was calculated as the product of force (after gravity correction by subtracting baseline force) and lever arm length (the distance between the knee-joint centre and the middle of the strap).

sEMG was recorded during pre and post measurement sessions with a wireless EMG system (Trigno; Delsys, Inc., Boston, MA). After shaving, abrading, and cleansing with $70 \%$ ethanol (standard preparation of 
the skin), single differential sensors (Delsys Inc., Boston, MA) were attached at six sites over the superficial quadriceps muscles using adhesive interfaces. sEMG sensors were located at the following percentages of thigh length (the distance from the knee-joint centre to the greater trochanter) above the superior border of the patella over the VM (25 and 35\%), VL (50 and 60\%) and RF (55 and 65\%). EMG signals were amplified and filtered at source $(x 300 ; 20-450-\mathrm{Hz}$ bandwidth) before further amplification (overall effective gain, x909) and subsequently sampled at $2,000 \mathrm{~Hz}$ using the same external A/D converter and computer software as the torque recordings. During the offline analysis, the sEMG data were time aligned with the torque signal due to an inherent 48-ms delay.

\section{Protocol for pre and post measurement sessions}

Testing sessions consisted of a series of sub-maximum warm-up contractions (the same as the training sessions) to prepare participants for the main tasks which were performed at five different knee-joint angles using their dominant leg. Each type of contraction was completed at all five joint angles (in either an ascending or descending order, randomised between both pre and both post measurement sessions), before moving on to the next type of contraction: MVCs; EVCs; evoked twitch contractions (see below).

\section{Maximal voluntary contractions}

Following a series of sub-maximum warm-up contractions (same as describe at the "training" above ) at the first measured angle, participants completed two MVCs at each of the five knee-joint angles. Participants were instructed to extend their knee and "push as hard as possible" for $\sim 4 \mathrm{~s}$ during MVCs with a $\geq 30 \mathrm{~s}$ recovery period between each MVC (at least $\sim 1$ min and $15 \mathrm{~s}$ ) and a minimum of 3 min rest between each angle. Biofeedback of the torque-time curve was displayed on a computer monitor in front of the participant during MVCs and a horizontal cursor was placed at the peak of the torque-time curve following the first MVC to encourage participants to exceed their best score. Intense verbal encouragement was also offered during all MVCs. During off-line analysis MVT at each angle was identified as the highest instantaneous torque during 
both MVCs, and the EMG amplitude of each individual EMG sensor at MVT was measured as the root mean square during a $500 \mathrm{~ms}$ time window (250 ms either side of MVT). EMG amplitude during MVT was subsequently normalized to a joint angle specific maximal M-wave peak-to-peak amplitude (MMAX P-P; see below) recorded from the corresponding EMG sensor during maximum twitch contractions, and these normalized values of each sensor were averaged to calculate whole quadriceps EMG at MVT (QEMGMVT).

\section{Explosive Voluntary Contractions}

Participants then performed 6-8 brief EVCs at each knee-joint. Prior to each contraction, participants were instructed to start from rest, without producing prior tension or countermovement, and on a verbal cue perform the contraction as "fast and hard" as possible for $\sim 1-\mathrm{s}$, attempting to reach $75 \% \mathrm{MVT}$ as quickly as possible and then relax. There was a 20 -s rest between EVCs at each angle, with a minimal time of $\sim 2$ min per angle, and a minimum of 5 min rest between angles. Baseline torque displayed on a sensitive scale was used to verify that pre-tension or countermovement did not occur before contraction onset and if either occurred the contraction was excluded. Torque onsets for EVC and evoked contractions (both twitch and octet) were identified manually by visual identification by a trained investigator using a systematic approach, as previously described (Tillin et al. 2013b). During off-line analysis, for each knee-joint angle and measurement session the two EVCs with the highest torque at $100 \mathrm{~ms}$ and peak torque $>70 \% \mathrm{MVT}$ were selected for further analysis. Explosive torque was measured at 50, 100, and $150 \mathrm{~ms}$ from torque onset $\left(\mathrm{T}_{50}, \mathrm{~T}_{100}\right.$ and $\mathrm{T}_{150}$ respectively) for each contraction and then a mean calculated for each angle during each session, before averaging across duplicate measurement sessions pre and post. Explosive torque also was calculated relative to MVT to identify if maximal and explosive strength changed proportionally from pre to post. sEMG amplitude (root mean square) of each individual sensor during EVCs was measured over epochs of 0-50, 0100 and 0-150 ms from sEMG onset and amplitudes were normalized to $\mathrm{M}_{\mathrm{MAX}} \mathrm{P}-\mathrm{P}$ from the corresponding sensor measured during twitch contractions. Subsequently normalized values of each sensor were averaged for each epoch to calculate $\left(\mathrm{QEMG}_{0-50}, \mathrm{QEMG}_{0-100}, \mathrm{QEMG}_{0-150}\right)$. sEMG onset was determined with a systemic manual method as previously described (Tillin et al. 2013b). 


\section{Evoked Twitch and Octet contractions}

After the EVCs and a 2-min rest, transcutaneous femoral nerve stimulation commenced and all evoked contractions were completed whilst the participant was voluntarily passive. An anode $(70$ x $100 \mathrm{~mm}$ carbon rubber electrode; Electro-Medical Supplies, Greenham, UK) was placed and secured over the greater trochanter and a cathode $(10 \mathrm{~mm}$ diameter, protruding $20 \mathrm{~mm}$ from a $35 \mathrm{x} 55 \mathrm{~mm}$ plastic base; Electro-Medical Supplies, Greenham, UK) was positioned over the femoral nerve in the femoral triangle, both coated in conductive gel. Electrical stimulation was then delivered with a constant-current variable-voltage stimulator (DS7AH, Digitimer Ltd., Welwyn Garden City, UK). At the first angle to be tested, the cathode was repositioned and a low-level current (40-60 mA) was delivered until an optimum site was identified based on the twitch torque response and then the cathode was secured with transpore tape. At each angle stimulation intensity was gradually increased until torque and the peak-to-peak amplitude of the M-wave plateaued. Thereafter, three further stimuli were delivered with a current of $150 \%$ of the plateau level to measure supramaximal $\mathrm{M}_{\mathrm{MAX}} \mathrm{P}-\mathrm{P}$ responses. An interval of $10 \mathrm{~s}$ was given between each twitch stimulus and a minimum of 2 min between angles. Twitch peak torque (Twitch PT), Twitch torque at $50 \mathrm{~ms}$ (Twitch $\mathrm{T}_{50}$ ), and $\mathrm{M}_{\mathrm{MAX}} \mathrm{P}-\mathrm{P}$ were averaged across the three supramaximal evoked contractions.

After all other procedures had been completed, octet contractions were performed during the second pre and first post measurement sessions at all joint angles. Octets were first evoked at progressive currents ( $\sim 15 \mathrm{~s}$ apart) until a plateau in the amplitude of peak torque and peak rate of torque development were achieved; this gradual increase in stimulation intensity procedure was only performed at the first angle tested. Then, two discrete pulse trains ( $\geq 15$ s apart) were delivered with a higher current $(\geq 20 \%$ above the plateau current to ensure supra-maximal stimulation) to evoke maximum octet contractions at each angle. Octets were performed in a counter-balanced order (i.e. half of the participants performed the octets in the order most flexed to most extended and the other half in the opposite order). Octet peak torque (Octet PT) and Octet torque at $50 \mathrm{~ms}$ (Octet $\mathrm{T}_{50}$ ) were measured as the mean across the two maximum octet contractions at each angle. The ratio of voluntary $\mathrm{T}_{50} /$ octet $\mathrm{T}_{50}$ was used as an additional measure of volitional neural efficacy (Hannah et al. 2012; 
Buckthorpe et al. 2012). A total of 3 participants (RT, $n=2$ and $\mathrm{CON}, n=1$ ) were unable to tolerate the discomfort associated with octet stimulation and did not perform this measurement.

\section{Statistical Analysis}

All data were anonymized prior to analysis. Reproducibility of all measurements over the 4-week intervention period were assessed by calculating the within-participant coefficient of variation of pre and post measurements [ $\mathrm{CV}_{\mathrm{W}}$; (SD/mean) x 100] for the CON group. Statistical analysis was performed after individual mean values had been averaged across duplicate test sessions at both time points (i.e. pre and post).

As a first step within-group changes were assessed with a two-way repeated measure ANOVA [time (pre vs. post) $\mathrm{x}$ angle $\left(35^{\circ}\right.$ vs. $50^{\circ}$ vs. $65^{\circ}$ vs. $80^{\circ}$ vs. $\left.95^{\circ}\right)$ ] for all variables and both groups. In order to thoroughly investigate the interaction effect (time $\mathrm{x}$ angle), and which specific angle presented higher changes after RT than another (i.e. within group joint angle specificity evidence), a within-group two-way repeated measure ANOVA [time (2) x angle (2)] analysis was also performed for pairwise combinations of angles (e.g. $35^{\circ}$ vs. $95^{\circ}$ etc.) with Bonferroni stepwise correction applied.

Subsequent, more rigorous analysis of between-group changes was only assessed if within-group analyses revealed a time or a time $\mathrm{x}$ angle effect within one of the groups. In that case, a two-way repeated measures ANOVA [group (2) vs. angle (5)], was performed with absolute change data (post-pre) to identify a main effect of group and group $\mathrm{x}$ angle interaction. If a group $\mathrm{x}$ angle interaction was found a subsequent twoway repeated measure ANOVA [group (2) x angle (2)] was also performed for pairwise combinations of angles (i.e. between group joint angle specificity evidence). Effect size (ES) was calculated as previously detailed (Cohen 1988) for within- and between-group comparisons, and classified as follows: $<0.20=$ "trivial"; $0.20-0.49=$ "small"; $0.50-0.79=$ "moderate" or $>0.80=$ "large". Statistical analysis was performed using SPSS version 23 (IBM Corporation, Armonk, New York, USA), the significance level was set at $\mathrm{P}<0.05$, and all data are reported as means $\pm \mathrm{SE}$ unless otherwise stated. 


\section{RESULTS}

\section{Group Characteristics at Baseline and Joint Angle Kinematics}

The two groups had similar age, body mass, and height $(\mathrm{P} \leq 0.752)$. When participant data were collapsed across angles at baseline, no differences $(\mathrm{P} \geq 0.271)$ were identified between groups (CON vs. RT) for MVT, explosive strength $\left(\mathrm{T}_{50}, \mathrm{~T}_{100}\right.$ or $\left.\mathrm{T}_{150}\right)$, evoked twitch torque, evoked octet torque, M MAx P-P amplitude, QEMGMvT and QEMG during EVC (absolute or normalized). Based on sagittal plane video recorded during the familiarisation session the actual knee joint angles during the plateau phase of MVCs at the five different positions were $33^{\circ} \pm 2^{\circ}, 50^{\circ} \pm 1^{\circ}, 62^{\circ} \pm 2^{\circ}, 78^{\circ} \pm 2^{\circ}, 91^{\circ} \pm 2^{\circ}$ at the five positions, with changes from rest of $\leq 4^{\circ}$ at all positions. Nonetheless we maintained the intended/prescribed joint angle positions as the terms for the five different positions i.e. $35^{\circ}, 50^{\circ}, 65^{\circ}, 80^{\circ}, 95^{\circ}$ throughout the experiment.

\section{Reproducibility of Torque and sEMG Measurements}

Reproducibility data were collapsed across all five angles. The within-participant reliability $\left(\mathrm{CV}_{\mathrm{W}}\right)$ of the strength measurements was as follows: MVT, $3.9 \%$; $\mathrm{T}_{150}, 7.8 \%$; $\mathrm{T}_{100}, 9.6 \%$; and $\mathrm{T}_{50}, 24.4 \%$. Normalized sEMG measurements presented a $\mathrm{CV}_{\mathrm{W}}$ of: $\mathrm{QEMG}_{\mathrm{MVT}}$ 9.8\%; $\mathrm{QEMG}_{0-150} 14.1 \%, \mathrm{QEMG}_{0-100} 16.1 \%$ and $\mathrm{QEMG}_{0-50}$ 19.8\%. Finally, evoked contractions presented $\mathrm{CV}_{\mathrm{W}}$ values of $4.5 \%$ for Octet $\mathrm{T}_{50}, 5.4 \%$ Octet $\mathrm{PT}$, $5.0 \%$ for Twitch $\mathrm{T}_{50}, 8.1 \%$ Twitch PT and $13.2 \%$ for M MAX P-P.

\section{Voluntary Torque}

\section{Maximum Voluntary Torque}

For RT within-group comparisons showed a main effect of time (time $\mathrm{x}$ angle ANOVA, $\mathrm{P}<0.001$ ) and post-hoc testing detected increases in MVT at the training angle $\left(65^{\circ},+12 \% \pm 2 \%\right)$, as well as at both of the adjacent angles $\left(80^{\circ},+7 \% \pm 2 \% ; 50^{\circ},+11 \% \pm 2 \%\right)$ and the most extended angle $\left(35^{\circ},+5 \% \pm 2 \%\right)$ after the intervention (All, Bonferroni pre vs. post $\mathrm{P} \leq 0.015$; ES $\geq 0.98$ "large" for $50^{\circ}$ and $65^{\circ}$; ES $\leq 0.37$ "small" for 
$35^{\circ}$ and $80^{\circ}$; Fig. $2 \mathrm{~A}$ ). After RT there was a time $\mathrm{x}$ angle interaction (time $\mathrm{x}$ angle ANOVA, $\mathrm{P}<0.001$ ) providing evidence of within-group joint angle specificity, with larger increases at the training angle and the two most adjacent angles compared to at least one other angle $\left(50^{\circ}>35^{\circ}\right.$ and $80^{\circ}, \mathrm{P} \leq 0.004 ; \mathrm{ES} \geq 1.26^{\text {"large"; }}$ $65^{\circ}>35^{\circ}, 80^{\circ}$ and $95^{\circ}, \mathrm{P} \leq 0.025 ; \mathrm{ES} \geq 1.47$ "large"; and $80^{\circ}>95^{\circ}, \mathrm{P}=0.009 ; \mathrm{ES}=0.79$ "moderate"). For $\mathrm{CON}$ there were no within-group time or interaction effects (time $\mathrm{x}$ angle ANOVA, $\mathrm{P}=0.406$; Fig. 2B).

Between-group comparisons for MVT change data (i.e. pre to post differences) showed a main effect of group (group $\mathrm{x}$ angle ANOVA, $\mathrm{P}=0.011$; i.e. overall training effect $\mathrm{RT}>\mathrm{CON}$ ). There was also a group $\mathrm{x}$ angle interaction (group $\mathrm{x}$ angle ANOVA, $\mathrm{P}=0.029$ ), indicating larger improvements after RT vs. CON that were dependent upon angle. Follow-up pairwise group x angle interactions revealed greater changes for RT than $\mathrm{CON}$ between both $65^{\circ}$ (training angle) vs. $95^{\circ}$ ( $\mathrm{P} \leq 0.010 ; \mathrm{ES} \geq 0.91$ "large"), and between $50^{\circ}$ vs. $95^{\circ}$ $(\mathrm{P} \leq 0.007 ; \mathrm{ES}=1.72$ "large" and $\mathrm{ES}=0.61$ "moderate", respectively). Thus providing the highest level of evidence for angle specificity [i.e. between group effects (vs. control) that differ according to angle].

\section{Explosive Voluntary Torque}

Within-group comparisons from pre to post for both RT and CON did not show a main effect of time or time $\mathrm{x}$ angle interaction effect for $\mathrm{T}_{50}$ or $\mathrm{T}_{100}$ (time $\mathrm{x}$ angle $\mathrm{ANOVA}, \mathrm{P} \geq 0.123$; Fig. 4). For $\mathrm{T}_{150}$ there was an increase (main effect of time) after RT, but not for CON (time x group ANOVAs: RT P=0.029; CON $\mathrm{P}=0.875$; Fig. 4A \& B). Post-hoc analysis of the $\mathrm{RT}$ group showed that $\mathrm{T}_{150}$ increased only at the training angle $\left(65^{\circ}\right)$ after RT (Bonferroni $\mathrm{P}=0.019 ; \mathrm{ES}=0.38$ “small”; Fig.4A). Between-group comparisons revealed no group or angle $\mathrm{x}$ group interaction effects for the pre-to-post changes in $\mathrm{T}_{150}$ (group $\mathrm{x}$ angle ANOVA, $\mathrm{P} \geq$ $0.227)$.

\section{Neuromuscular Activation and MMax Amplitude}

Within-group comparisons of normalized QEMG MVT $_{\text {(\%MAX }}$ P-P) had a main effect of time after RT (time $\mathrm{x}$ angle ANOVA, $\mathrm{P}=0.004$ ) but this was not the case for $\mathrm{CON}$ (time $\mathrm{x}$ group ANOVA, $\mathrm{P}=0.382$ ). No 
interaction was found for both groups (time $\mathrm{x}$ angle ANOVA, $\mathrm{P} \geq 0.502$ ). For RT subsequent post-hoc testing revealed normalized $\mathrm{QEMG}_{\mathrm{MVT}}$ increased at $35^{\circ}(+8 \% \pm 3 \%), 50^{\circ}(+19 \% \pm 5 \%)$ and $65^{\circ}(+18 \% \pm 5 \%$; Bonferroni $\mathrm{P} \leq 0.031$; $\mathrm{ES}=0.27$ "small” for $35^{\circ}$ or $\mathrm{ES} \geq 0.54$ "moderate" for $50^{\circ}$ and $65^{\circ}$; Fig. $\left.2 \mathrm{D}\right)$, but not $80^{\circ}$ or $95^{\circ}$. Between-group comparisons revealed a main effect of group (group x angle ANOVA, $\mathrm{P}=0.006$ i.e. greater increases in activation after RT than $\mathrm{CON}$ ), but not an angle $\mathrm{x}$ group interaction effect for normalized $\mathrm{QEMG}_{\mathrm{MVT}}$ (group x angle ANOVA, $\mathrm{P}=0.587$ ).

Within-group comparisons for normalized QEMG during EVCs $\left(\mathrm{QEMG}_{0-50}, \mathrm{QEMG}_{0-100}, \mathrm{QEMG}_{0-150}\right)$ did not show time or time $\mathrm{x}$ angle effects in either group RT or CON (time $\mathrm{x}$ angle ANOVA, $\mathrm{P} \geq 0.233$ ). Within-group measures of $\mathrm{M}_{\mathrm{MAX}} \mathrm{P}-\mathrm{P}$ amplitude did not show a main effect for time or an interaction effect for either RT or CON (time x group ANOVA, $\mathrm{P} \geq 0.681$ ).

\section{Intrinsic Contractile Properties}

\section{Absolute Torque}

Within-group comparisons for both the RT and CON groups revealed no main effect of time or time $\mathrm{x}$ angle interactions for evoked measures (Twitch $\mathrm{T}_{50}$, Twitch PT, Octet $\mathrm{T}_{50}$; time $\mathrm{x}$ angle ANOVA, $\mathrm{P} \geq 0.142$ ). However, the RT group, but not CON, presented a main effect of time for Octet PT (time x angle ANOVA, $\mathrm{P}=0.014$ and 0.245 , respectively). Post-hoc testing revealed Octet PT increased after $\mathrm{RT}$ at $65^{\circ}$ and $50^{\circ}$ (Bonferroni pre vs. post $\mathrm{P} \leq 0.037 ; \mathrm{ES} \leq 0.026$ "small”). Between-group comparisons of Octet PT change data showed no main effect time or group $\mathrm{x}$ angle interaction effect (group $\mathrm{x}$ angle ANOVA, $\mathrm{P} \geq 0.440$ ).

\section{DISCUSSION}

The primary aim of this investigation was to determine if joint angle specific changes in strength were a genuine systematic effect, and whether changes in neuromuscular activation might explain any angle specific strength changes. RT performed at a mid-range knee-joint angle $\left(65^{\circ}\right.$ the angle of peak torque) demonstrated increases in maximal strength at four out of five angles, and the changes at some angles, specifically the 
training angle and the adjacent more extended angle $\left(65 \& 50^{\circ}\right)$, were larger than other positions, demonstrating within-group evidence of joint angle specificity. This study also found robust, between-group evidence of joint angle specificity with larger improvements after RT at some angles $\left(65 \& 50^{\circ}\right)$ than others $\left(95^{\circ}\right)$ in comparison to CON. Joint angle specificity was thus confirmed using rigorous experimental design and analysis. Neuromuscular activation showed within group increases (effects of time) at the training angle and more extended positions $\left(35,50\right.$ and $\left.65^{\circ}\right)$ only for RT, whilst these changes were largely coincident with the strength changes (increases at the same angles and within the same group) there was no within- or betweengroup evidence of joint angle specificity for neuromuscular activation. Moreover, the addition of ECT to the training session did not improve the early phase of the explosive torque production.

As expected between-group comparisons revealed significant effects of group and thus RT on changes in maximum strength and associated neural activation, reinforcing the efficacy of short-term RT for these outcomes. Numerous contemporary studies have also found enhanced quadriceps sEMG as an index of neuromuscular activation, post RT [e.g. (Tillin et al. 2012; de Ruiter et al. 2012; Balshaw et al. 2016)]. Whilst, the aim of the study was not to investigate the precise neural mechanisms whereby the changes in neuromuscular activation occur, increases in neuromuscular activation after a RT intervention have largely been attributed to increases in motor unit recruitment/synchronization, decrease in recruitment threshold, increases in the firing frequency/discharge rate and/or decrease in neural inhibition (Sale 1988; Gabriel et al. 2006; Folland and Williams 2007; Del Vecchio et al. 2019).

However, the main focus of the current experiment was the joint angle specificity of these improvements in strength and neuromuscular activation. Within-group analyses showed that RT improved MVT at the training angle $(\sim 12 \%)$ and at both adjacent angles and the most extended angles. This indicates that improvements at the training angle transfer to angles $>15^{\circ}$ of flexion (longer lengths) and $>30^{\circ}$ of extension (shorter lengths) from the training angle. Previous work has also suggested greater transfer of isometric strength gains at the training angle to more extended (shorter) than flexed (longer) positions (Weir et al. 1995; Noorkoiv et al. 2014). Moreover, joint angle specificity was demonstrated within the RT group, with MVT at the training and adjacent angles increasing compared to at least one other angle, and also by 
more rigorous between-group comparisons showing larger improvements after RT at some angles $\left(65 \& 50^{\circ}\right)$ than others $\left(95^{\circ}\right)$ while no changes were detected at CON. Therefore, this study provides rigorous evidence that the joint angle specificity of isometric RT is a genuine effect.

The present study detected increases in agonist neuromuscular activation at MVT (i.e. normalized $\left.\mathrm{QEMG}_{\mathrm{MVT}}\right)$ after RT at the training angle and more extended knee positions (7-19\%, within-group), that is at 3 of the 4 positions where the strength gains also occurred in this group, with no changes in strength or activation after CON. These changes in activation would seem unlikely to have occurred at the same angles and in the same group as for the strength gains by chance, and therefore provide a ready explanation for the increases in maximum strength at these angles. However, we found no within- or between-group evidence of joint angle specificity for neuromuscular activation. Previous studies investigating the knee-joint angle specificity of RT have detected similar increases in neuromuscular activation after training (Kubo et al. 2006; Noorkoiv et al. 2014) but also no joint angle specificity for neuromuscular activation. Neuromuscular activation in this study was assessed with SEMG amplitude normalized to $\mathrm{M}_{\mathrm{MAX}}$, which is known to reduce the confounding effects of electrode relocation and subcutaneous tissue thickness (Lanza et al. 2018). Nonetheless sEMG measurements at MVT clearly showed greater between- and within- participant variability than measurements of MVT and this likely impaired the possibility to detect angle specific training adaptations in neuromuscular activation.

The transfer of neuromuscular activation across knee-joint angles does not really has a direct explanation but a several possible mechanisms. For instance, it is known that the cortical exitability, modulated by the motor cortex (Carroll et al. 2011), is influenced by joint angle (Mitsuhashi et al. 2007; Chye et al. 2010). Thus, the adaptations of the training intervention at the present study could have occurred at supraspinal level at the trained angle and reflected as well in the other knee-joint angles. Additionally, hamstrings coactivation changes across knee-joint angles (Kubo et al. 2004; Lanza et al. 2017) and may have altered after the training intervention and reflected in our results. Moreover, mechanically, the cross-brigde binding is not optimall at more extended or flexed positions (Rassier et al. 1999), which may also influence the adpations after training with higher responses where the binding is optimal. 
In the present study there was a modest increase in explosive strength only during the late phase of contraction $(150 \mathrm{~ms})$ after $\mathrm{RT}$, and only at the training angle $(\sim 7 \%)$ i.e. no transfer to other angles. Subsequently there was no evidence of joint angle specific adaptations in explosive strength. Whilst there were no changes in neuromuscular activation during explosive torque production after RT, Octet PT did increase by $\sim 4.3 \%$ at $65^{\circ}$ and $50^{\circ}$ for the RT group only. This change in evoked peak torque indicates a modest morphological adaptation, most likely hypertrophy, may have occurred even within the 4 weeks of this intervention, similar to previous reports of subtle hypertrophic effects within only a few weeks of RT (Seynnes et al. 2007; Buckthorpe et al. 2012; Maeo et al. 2018). Furthermore muscle size is known to be a determinant of late phase explosive strength (Erskine et al. 2014; Evangelidis et al. 2017) and thus could account for the modest increase in explosive $\mathrm{T}_{150}$.

Our previous work found quite marked training specificity effects of sustained vs. explosive contraction RT; with greater increases in early phase explosive torque after ECT vs. SCT and greater increases in MVT after SCT vs. ECT (Balshaw et al. 2016). In this study both types of contractions (ECT and SCT) were combined to see if adaptations in both explosive and maximum strength would occur. However, whilst the combined training elicited increased MVT, as we have previously found for SCT (Tillin and Folland 2014; Balshaw et al. 2016) it did not increase explosive torque at any time point during the rising/explosive phase of contraction. Thus the combined training used in this study resulted in changes in function, maximum and explosive strength, similar to SCT alone (Tillin and Folland 2014; Balshaw et al. 2016), with no improvement in early phase explosive strength during the first $100 \mathrm{~ms}$ of contraction. In other words the unique functional adaptation to ECT, improved early phase explosive strength, appears to be negated when combined with SCT within the same training session, perhaps suggestion some interference effect of the combined training. This finding would benefit from replication in a study where the primary focus is the type and combination of RT contractions.

The present study has limitations and strengths that are worth highlighting. One limitation was that in order to carefully consider the transfer to adjacent joint angles the positions assessed in this study were $\pm 30^{\circ}$ of the training angle and thus only $60^{\circ}$, rather than the whole range of knee-joint of movement. The volume 
of measurements in the current study was high with maximum and explosive voluntary contractions performed at each of 5 angles within each measurement session, and this appears to have reduced the reproducibility of the measurements compared to our previous experiments with strength measurements at just one angle [e.g. (Balshaw et al. 2016)]. However, the current study involved averaging data across duplicate measurements sessions both pre and post the intervention period in order to further improve reproductibilty, and these sessions involved a counterbalanced angle order to reduce the possibility of systematic order effects.

\section{CONCLUSION}

In conclusion, after RT maximal strength increased at 4 out of 5 knee-joint angles and we found novel and unique evidence of joint angle specificity; larger improvements after RT at some angles than others in comparison to CON. Increases in neuromuscular activation occurred at 3 of the same joint angles as strength gains for the RT group, but not control, giving some support to the notion of increased activation underpinning angle specific strength gains. However, importantly there was no evidence of joint angle specific changes in activation either from within- or between-group analyses, likely due to the variability in the sEMG adaptatons. Finally, the combination of explosive and sustained contractions during each training session did not result in early phase explosive strength gains during a 4-week training period. 


\section{REFERENCES}

Alegre LM, Ferri-Morales A, Rodriguez-Casares R, Aguado X (2014) Effects of isometric training on the knee extensor moment-angle relationship and vastus lateralis muscle architecture. Eur J Appl Physiol 114:2437-2446 . doi: 10.1007/s00421-014-2967-x

Balshaw TG, Fry A, Maden-Wilkinson TM, et al (2017) Reliability of quadriceps surface electromyography measurements is improved by two vs. single site recordings. Eur J Appl Physiol 117:1085-1094 . doi: $10.1007 / \mathrm{s} 00421-017-3595-\mathrm{z}$

Balshaw TG, Massey GJ, Maden-Wilkinson TM, et al (2016) Training-specific functional, neural, and hypertrophic adaptations to explosive- vs. sustained-contraction strength training. J Appl Physiol 120:1364-1373 . doi: 10.1152/japplphysiol.00091.2016

Behan FP, Pain MTG, Folland JP (2018) Explosive voluntary torque is related to whole-body response to unexpected perturbations. J Biomech 81:86-92 . doi: https://doi.org/10.1016/j.jbiomech.2018.09.016

Buckthorpe MW, Hannah R, Pain TG, Folland JP (2012) Reliability of neuromuscular measurements during explosive isometric contractions, with special reference to electromyography normalization techniques. Muscle Nerve 46:566-576 . doi: 10.1002/mus.23322

Carroll TJ, Selvanayagam VS, Riek S, Semmler JG (2011) Neural adaptations to strength training: Moving beyond transcranial magnetic stimulation and reflex studies. Acta Physiol 202:119-140 . doi: 10.1111/j.1748-1716.2011.02271.x

Chye L, Nosaka K, Murray L, et al (2010) Corticomotor excitability of wrist flexor and extensor muscles during active and passive movement. Hum Mov Sci 29:494-501 . doi: 10.1016/j.humov.2010.03.003

Cohen J (1988) Statistical power analysis for the behavioral sciences. Stat. Power Anal. Behav. Sci. 2nd:567

de Ruiter CJ, Hutter V, Icke C, et al (2012) The effects of imagery training on fast isometric knee extensor torque development. J Sports Sci 30:166-174 . doi: 10.1080/02640414.2011.627369

de Ruiter CJ, Kooistra RD, Paalman MI, de Haan A (2004) Initial phase of maximal voluntary and electrically stimulated knee extension torque development at different knee angles. J Appl Physiol 97:1693-1701

Del Vecchio A, Casolo A, Negro F, et al (2019) The increase in muscle force after 4 weeks of strength training is mediated by adaptations in motor unit recruitment and rate coding. J Physiol 0:1-15 . doi: 
Erskine RM, Fletcher G, Folland JP (2014) The contribution of muscle hypertrophy to strength changes following resistance training. Eur J Appl Physiol 114:1239-1249 . doi: 10.1007/s00421-014-2855-4

Evangelidis PE, Massey GJ, Ferguson RA, et al (2017) The functional significance of hamstrings composition: is it really a "fast" muscle group? Scand J Med Sci Sports 27:1181-1189 . doi: $10.1111 / \mathrm{sms} .12786$

Folland JP, Williams AG (2007) The Adaptations to Strength Training. Sport Med 37:145-168 . doi: $10.2165 / 00007256-200737020-00004$

Gabriel DA, Kamen G, Frost G (2006) Neural Adaptations to Resistive Exercise. Sport Med 36:133-149 . doi: 10.2165/00007256-200636020-00004

Gardner GW (1963) Specificity of Strength Changes of the Exercised and Nonexercised Limb following Isometric Training. Res Quarterly Am Assoc Heal Phys Educ Recreat 34:98-101 . doi: $10.1080 / 10671188.1963 .10613225$

Hannah R, Minshull C, Buckthorpe MW, Folland JP (2012) Explosive neuromuscular performance of males versus females. Exp Physiol 97:618-629 . doi: 10.1113/expphysiol.2011.063420

Izquierdo M, Aguado X, Gonzalez R, et al (1999) Maximal and explosive force production capacity and balance performance in men of different ages. Eur J Appl Physiol Occup Physiol 79:260-267 . doi: $10.1007 / \mathrm{s} 004210050504$

Kinser PA, Robins JL (2013) Control Group Design: Enhancing Rigor in Research of Mind-Body Therapies for Depression. Evidence-Based Complement Altern Med 2013:1-10 . doi: 10.1155/2013/140467

Kitai TA, Sale DG (1989) Specificity of joint angle in isometric training. Eur J Appl Physiol Occup Physiol $58: 744-8$

Kubo K, Ohgo K, Takeishi R, et al (2006) Effects of isometric training at different knee angles on the muscle-tendon complex in vivo. Scand J Med Sci Sport 16:159-167 . doi: 10.1111/j.16000838.2005.00450.x

Kubo K, Tsunoda N, Kanehisa H, Fukunaga T (2004) Activation of agonist and antagonist muscles at different joint angles during maximal isometric efforts. Eur J Appl Physiol 91:349-52 . doi: $10.1007 / \mathrm{s} 00421-003-1025-\mathrm{x}$

Lanza MB, Balshaw TG, Folland JP (2017) Do changes in neuromuscular activation contribute to the knee 
extensor angle-torque relationship? Exp Physiol 102:962-973 . doi: 10.1113/EP086343

Lanza MB, Balshaw TG, Massey GJ, Folland JP (2018) Does normalization of voluntary EMG amplitude to MMAX account for the influence of electrode location and adiposity? Scand J Med Sci Sports 28:2558-2566 . doi: 10.1111/sms.13270

Lum D, Barbosa TM (2019) Brief Review: Effects of Isometric Strength Training on Strength and Dynamic Performance. Int J Sports Med 40:363-375 . doi: 10.1055/a-0863-4539

Maeo S, Shan X, Otsuka S, et al (2018) Neuromuscular Adaptations to Work-matched Maximal Eccentric versus Concentric Training. Med Sci Sport Exerc 50:1629-1640 . doi: 10.1249/MSS.0000000000001611

Maffiuletti NA, Aagaard P, Blazevich AJ, et al (2016) Rate of force development: physiological and methodological considerations. Eur J Appl Physiol 116:1091-1116 . doi: 10.1007/s00421-016-3346-6

Mitsuhashi K, Seki K, Akamatsu C, Handa Y (2007) Modulation of Excitability in the Cerebral Cortex Projecting to Upper Extremity Muscles by Rotational Positioning of the Forearm. Tohoku J Exp Med 212:221-228 . doi: 10.1620/tjem.212.221

Morganti CM, Nelson ME, Fiatarone MA, et al (1995) Strength improvements with $1 \mathrm{yr}$ of progressive resistance training in older women. Med Sci Sports Exerc 27:906-912

Noorkoiv M, Nosaka K, Blazevich AJ (2014) Neuromuscular adaptations associated with knee joint anglespecific force change. Med Sci Sports Exerc 46:1525-1537 . doi: 10.1249/MSS.0000000000000269

Paasuke M, Ereline J, Gapeyeva H (2001) Knee extension strength and vertical jumping performance in nordic combined athletes. J Sports Med Phys Fitness 41:354-361

Pijnappels M, van der Burg (Petra) J C E, Reeves ND, van Dieën JH (2008) Identification of elderly fallers by muscle strength measures. Eur J Appl Physiol 102:585-592 . doi: 10.1007/s00421-007-0613-6

Rassier DE, MacIntosh BR, Herzog W (1999) Length dependence of active force production in skeletal muscle. J Appl Physiol 86:1445-1457

Sale DG (1988) Neural adaptation to resistance training. Med Sci Sports Exerc 20:S135-45

Seynnes OR, de Boer M, Narici M V. (2007) Early skeletal muscle hypertrophy and architectural changes in response to high-intensity resistance training. J Appl Physiol 102:368-373 . doi:

10.1152/japplphysiol.00789.2006 
Thepaut-Mathieu C, Van Hoecke J, Maton B (1988) Myoelectrical and mechanical changes linked to length specificity during isometric training. J Appl Physiol 64:1500-1505

Tillin NA, Folland JP (2014) Maximal and explosive strength training elicit distinct neuromuscular adaptations, specific to the training stimulus. Eur J Appl Physiol 114:365-374 . doi: 10.1007/s00421013-2781-x

Tillin NA, Pain MTG, Folland J (2013a) Explosive force production during isometric squats correlates with athletic performance in rugby union players. J Sports Sci 31:66-76 . doi:

$10.1080 / 02640414.2012 .720704$

Tillin NA, Pain MTG, Folland JP (2012) Short-term training for explosive strength causes neural and mechanical adaptations. Exp Physiol 97:630-641 . doi: 10.1113/expphysiol.2011.063040

Tillin NA, Pain MTG, Folland JP (2013b) Identification of contraction onset during explosive contractions. Response to Thompson et al. "Consistency of rapid muscle force characteristics: Influence of muscle contraction onset detection methodology" [J Electromyogr Kinesiol 2012;22(6):893-900]. J Electromyogr Kinesiol 23:991-994 . doi: 10.1016/j.jelekin.2013.04.015

Ullrich B, Kleinöder H, Brüggemann GP (2009) Moment-angle relations after specific exercise. Int J Sports Med. doi: 10.1055/s-0028-1104589

Weir JP, Housh TJ, Weir LL, Johnson GO (1995) Effects of unilateral isometric strength training on joint angle specificity and cross-training. Eur J Appl Physiol Occup Physiol 70:337-343 . doi: 10.1007/BF00865031 
Fig. 1 A schematic of the hip and knee angles during the maximum, explosive, and evoked knee extension contractions performed during the testing sessions and the training angle used during the training sessions. Arrows indicate ascending and descending order which the participant performed the tasks in a counterbalanced order within pre and post-test sessions. If during the first test session the participant performed the task in a descending order for the next test session a descending order was performed.

Fig. 2 Knee extensor MVT-angle relationships and normalized QEMG-angle relationships, at knee-joint angles of $35^{\circ}, 50^{\circ}, 65^{\circ}, 80^{\circ}$ and $95^{\circ}$. Pre- and post-resistance training (RT; A and C) and control (CON; B and D) interventions. Brackets indicate within-group effects of time (pre vs. post, post-hoc Bonferoni; $\mathrm{P}<0.05$ ) for both MVT and normalized QEMG $\mathrm{MVT}_{\mathrm{M}}$ after RT. Symbols indicate within-group angle $\mathrm{x}$ time interactions; specific angles with larger increases after RT than other angles: * increases $>$ than $35^{\circ}, 80^{\circ}$ and $95^{\circ}$; \# increases $>$ than $35^{\circ}$ and $95^{\circ} ; \S>$ increases than $95^{\circ}$ (post-hoc pairwise two-way ANOVA of angle $\mathrm{x}$ time, $\mathrm{P}<0.05)$. Data are means $\pm \mathrm{SE}(\mathrm{RT}, n=13 ; \mathrm{CON}, n=9)$.

Fig. 3 Absolute change $(\Delta)$ in MVT (A) and normalized QEMGMVT (B) at knee-joint angles of $35^{\circ}, 50^{\circ}, 65^{\circ}$, $80^{\circ}$ and $95^{\circ}$ between pre and post for resistance training (RT) and control (CON) groups after a 4-week intervention period. Between-group ANOVA interaction effects (angle x group) were found for $\triangle \mathrm{MVT}$ as denoted by the following symbols: * larger difference for RT vs CON for $65^{\circ}$ vs and $95^{\circ}$; $\S$ larger difference for $\mathrm{RT}$ vs $\mathrm{CON}$ for $50^{\circ}$ vs $95^{\circ}$. Data are means $\pm \mathrm{SE}(\mathrm{RT}, n=13$; $\mathrm{CON}, n=9$ ).

Fig. 4 Knee extensor explosive torque-angle relationships at knee-joint angles of $35^{\circ}, 50^{\circ}, 65^{\circ}, 80^{\circ}$ and $95^{\circ}$ and measured at three time points $50 \mathrm{~ms}\left(\mathrm{~T}_{50}\right), 100 \mathrm{~ms}\left(\mathrm{~T}_{100}\right)$ and $150 \mathrm{~ms}\left(\mathrm{~T}_{150}\right)$ from torque onset for resistance training (RT; Fig. 4A) and control (CON; Fig. 4B) groups pre and post a 4-week intervention period. Differences between group pre and post intervention was only detected for RT at $150 \mathrm{~ms}$ (pre vs post, posthoc Bonferoni, $\mathrm{P}=0.029)$. Symbol * indicate post- higher than pre-training $(\mathrm{P}=0.019)$. Data are means $\pm \mathrm{SD}$ (RT, $n=13 ; \mathrm{CON}, n=9)$. 


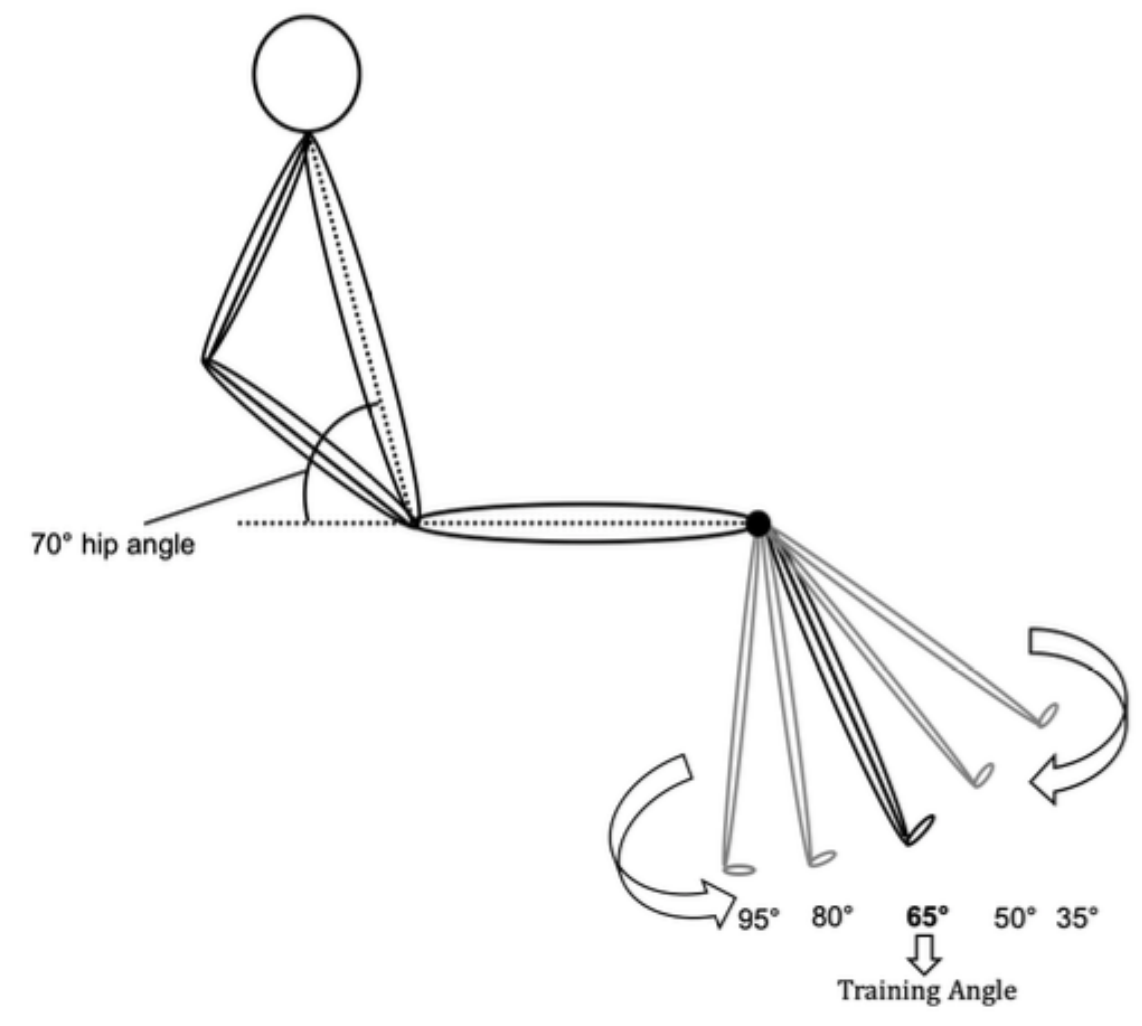

Fig.1. 

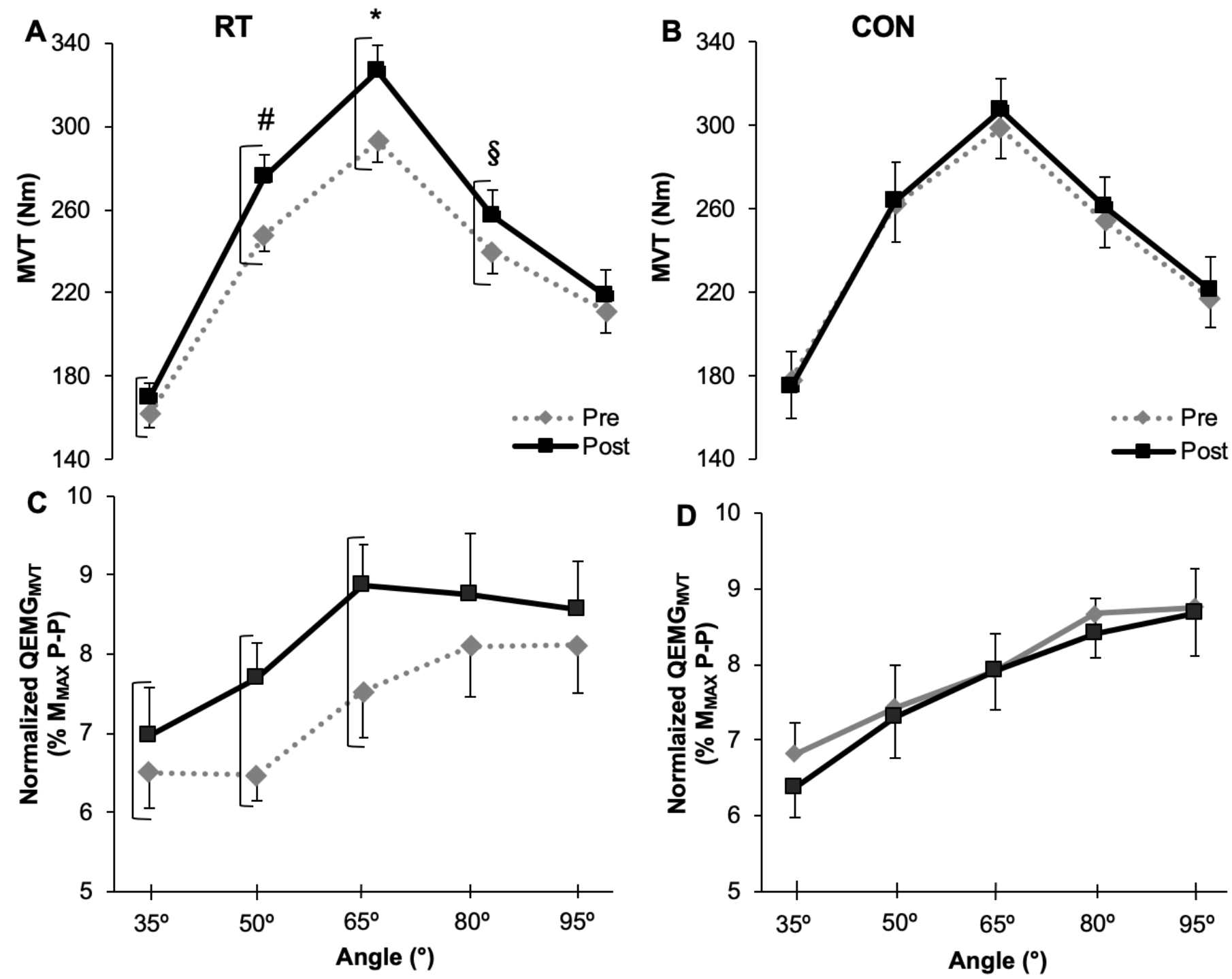

Fig. 2. 

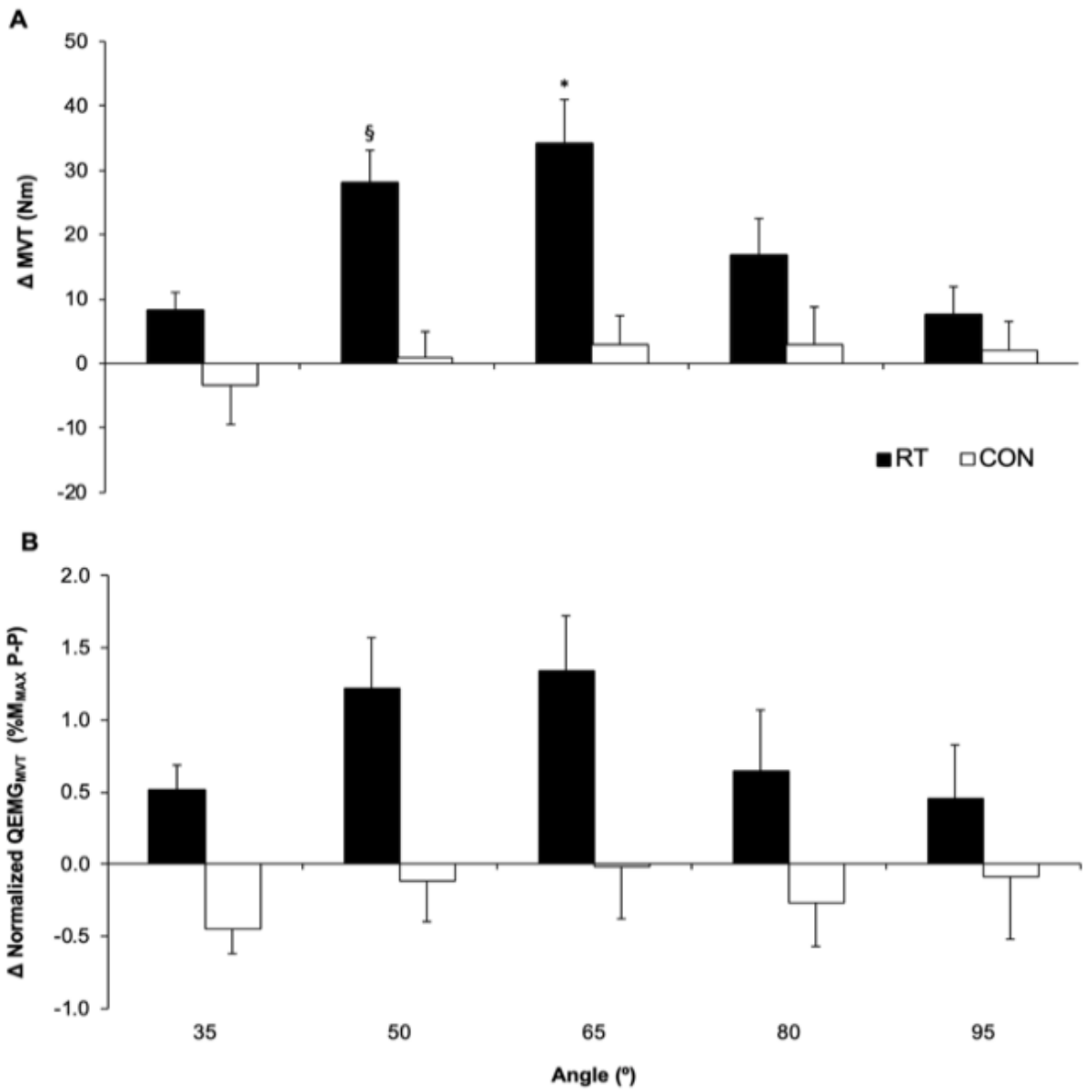

Fig.3. 

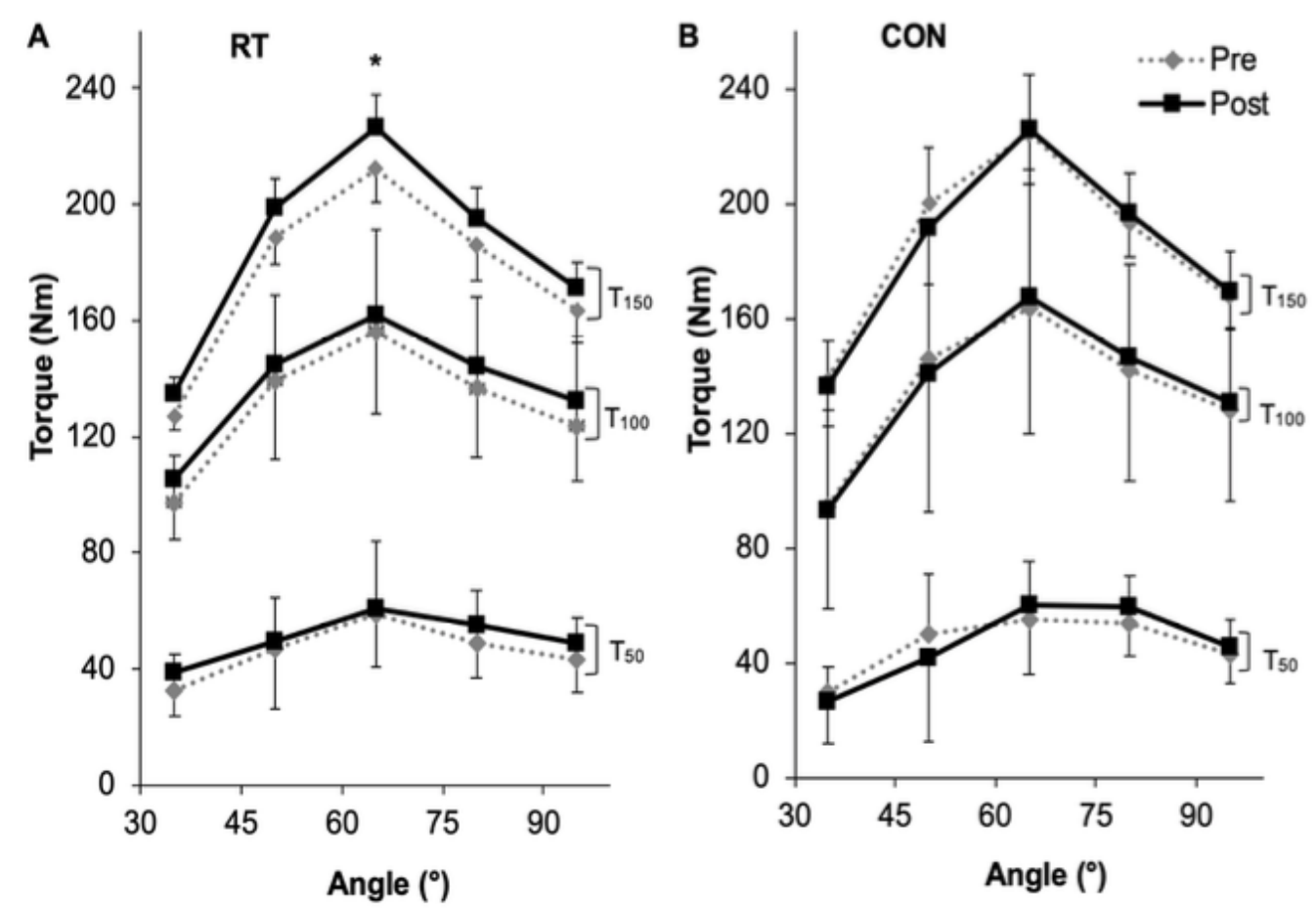

Fig.4. 